\title{
Particularities of pediatric systemic lupus erythematosus - literature review
}

\author{
Oana-Maria Farkas ${ }^{1,2}$, Sigrid Covaci ${ }^{1,3,4}$, Alexis-Virgil Cochino ${ }^{1,3}$ \\ 1"Alessandrescu-Rusescu" National Institute for Mother and Child Health, Bucharest, Romania \\ 2"Dr. I. Cantacuzino" University Hospital, Bucharest, Romania \\ 3"Carol Davila" University of Medicine and Pharmacy, Bucharest, Romania \\ 4"Sf. Maria" University Hospital, Bucharest, Romania
}

\begin{abstract}
Pediatric Systemic Lupus Erythematosus (pSLE) is a complex autoimmune disease with onset of symptoms before 18 years of age, accounting for $18-20 \%$ of all SLE cases. Although the American College of Rheumatology (ACR) classification criteria and the SLICC (Systemic Lupus International Collaborating Clinics) classification criteria for adults with SLE are commonly applied to pSLE, its clinical onset is different. Renal and neurological involvement tend to be more common and more severe in pediatric population as compared to adults, being therefore major determinants of prognosis and mortality. Renal biopsy should be performed as early as possible in every case of pSLE with signs and symptoms of renal impairment.
\end{abstract}

Keywords: pediatric, systemic lupus erythematosus, particularities

\section{INTRODUCTION}

Pediatric Systemic Lupus Erythematosus (pSLE) is a complex autoimmune disease which can involve any organ system, with onset of symptoms before 18 years of age, accounting for $18-20 \%$ of all cases of SLE (1). Most cases occur after the age of 10 , with a female prevalence which increases with age (sex ratio F:M 4:3 before puberty, 4:1 afterwards) $(2,3,6)$.

\section{CLINICAL FEATURES}

Children and adolescents develop a more severe disease than adults, with renal and neurological involvement (3). In pSLE the onset pattern and various clinical features' frequency are different from adults. The most important differences in disease activity between pSLE and adult SLE (aSLE) are lupus nephritis and neuropsychiatric lupus.

Constitutional symptoms (fever, fatigue, myalgias, anorexia, weight loss) are common in pSLE onset. Unexplained fever and lymphadenopathy appear with higher frequency in pSLE (6).
TABLE 1. Clinical features of $p S L E$

Cons tu onal: fever, involuntary weight loss
Hepato/splenomegaly, lymphadenopathy
Cutaneous: malar rash, discoid rash, subacute lesions,
vasculi s, alopecia, petechiae
Photosensi vity
Mucosal lesions: petechiae, oral ulcera ons (generally
painless)
Musculoskeletal: arthri s/arthralgia
Renal: edema, hematuria
Neurologic: headache, psychosis, cogni ve dysfunc on,
depression, cerebrovascular disease, seizures etc
Cardiovascular: pericardi s, myocardi s
Pulmonary: pleuri s, acute lupus pneumonia (fever, dyspnea,
coughing, hemoptysis), pulmonary hemorrhage, pulmonary
hypertension

A study comparing 56 children with juvenile onset SLE and 194 patients with adult onset SLE, found that renal involvement, encephalopathy and hemolytic anemia, were significantly more common in juvenile SLE as compared to adult SLE (4). In other studies, cutaneous vasculitis, nephropathy and seizures were more frequent in pSLE, whereas articular manifestations had a lower frequency in children $(5,6)$. There may be more arthritis with pediat- 
ric SLE, while arthralgias and myalgias appear to be more frequently encountered in aSLE (6). Inflammatory rashes and photosensitivity seem to be more frequent in pSLE in some studies, but in others have the same frequency as in adult onset SLE. In all studies discoid lesions are less common in children.

Nephritis is the most common major organ involvement, often present at diagnosis and manifested by peripheral edema, microscopic/macroscopic hematuria. Nephritis is more common in children as compared to adults, with studies showing that over $50 \%$ (in some studies even $75-80 \%$ ) of children develop clinically evident nephritis at some point of their illness $(2,3,4)$. In the largest published cohort, which included 795 adult-onset and 90 childhoodonset lupus cases, with a follow-up of 16 and 13 years, respectively, $56 \%$ of children versus $37 \%$ of adults developed renal disease. Nephritis tends to be not only more frequent, but also more severe in pSLE which makes it the most important determinant in the prognosis of the disease. Therefore renal biopsy should be performed in all pediatric patients with signs and symptoms of nephritis preferably within the first month after disease onset (7). In both pSLE and aSLE, diffuse proliferative glomerulonephritis is the most frequent abnormality (present in $40-75 \%$ of cases). Proteinuria (60-70\%) is the most common manifestation of renal disease, followed by microscopic hematuria (40-50\%), hypertension (25\%) and compromised renal function (8).

Neuropsychiatric lupus (NP-SLE) varies according to the diagnostic criteria, but it seems to be more frequent in pSLE $(6,8)$. Depression is the most common mood disorder in children and in adults, but psychosis, chorea and encephalopathy occurs preferentially with pSLE (6). Psychosis with visual hallucinations is a hallmark of NP-SLE involvement in pediatric patients, with seizures and antiphospholipid antibody-associated unilateral chorea being the most prevalent neurological signs (8). It seems that the ACR criteria regarding aSLE are not suited to pSLE (6).

Uncommon manifestations are more prevalent in pSLE, such as gastrointestinal events with intestinal vasculitis and pancreatitis or autoimmune liver disease (8).

\section{LABORATORY FINDINGS}

The most common hematologic manifestation in children is mild leukopenia $\left(3,000-4,000\right.$ cells $\left./ \mathrm{mm}^{3}\right)$,

\section{TABLE 2. Laboratory features in pSLE}

Hematological: anemia, leukopenia, thrombocytopenia Biological inflammatory syndrome: important ESR eleva on, but with normal CRP

Urinalysis: proteinuria, hematuria and cellular casts Coagula on anomalies

Autoan bodies:

- Antinuclear antibodies (ANA - present in over $95 \%$ of pediatric patients)

- anti-double-stranded DNA (anti-dsDNA)

- anti Smith antibodies (anti Sm)

- antiphospholipid antibodies (lupus anticoagulant, anticardiolipin antibodies, anti beta2-glycoprotein 1, false positive VDRL)

Immunological anomalies:

- hypergammaglobulinemia

- crioglobulinemia

- low serum complement levels

- elevated immune circulating complexes

- elevated rheumatoid factor

- Positive Coombs test

usually due to lymphopenia $\left(<1,500\right.$ cells $\left./ \mathrm{mm}^{3}\right)$. While persistent lymphopenia, which may be a feature of active disease, is correlated with anti-dsDNA antibodies levels and is associated with NPSLE and mucocutaneous involvement in pSLE and aSLE, neutropenia is more frequently a result of treatment $(6,9)$. In pSLE, anemia is usually mild to moderate and can take any form: anemia of chronic disease (normocytic and normochromic), iron deficiency anemia, or a Coombs' positive hemolytic anemia. The most common cause of anemia in both pSLE and aSLE is chronic disease. Hemolytic anemia is more prevalent in pSLE than aSLE as it is suggested by several studies (8). Overall, the prevalence of thrombocytopenia appears to be somewhat higher in pSLE than aSLE, being mild $(<150,000)$ to profound $(<10,000)$. Children and adolescents with chronic ITP should be assessed for the presence of ANA for the risk of developing SLE.

The prevalence of anticardiolipin (aCL) antibodies and lupus anticoagulant (LAC) appears similar in groups of adults and children with SLE (6). Most studies have shown no difference in the prevalence of ANA between adult and pediatric onset lupus patients (8), but they suggest that elevated levels of anti-dsDNA antibodies are more common in pSLE than in aSLE (6). Low C3 levels also appear to be more prevalent in childhood-onset lupus.

\section{CONCLUSIONS}

Children and adolescents develop more severe SLE than adults, with renal and neurological involvement. Nephritis is more common but also more 
severe in pSLE in children as compared to adults, which makes it the most important determinant in disease prognosis. Therefore, renal biopsy should be performed in all pediatric patients with signs and symptoms of nephritis. Neuropsychiatric lupus seems to be more frequent in pSLE. There is no dif-

\section{REFERENCES}

1. Kamphuis S., Silverman E.D. Prevalence and burden of pediatric-onset systemic lupus erythematosus, Nat Rev Rheumatol 6:538-546, 2010

2. Ronald M. Laxer, Susanne M. Benseller in Kelley's Textbook of Rheumatology. Philadelphia, PA: Elsevier/Saunders, chapter 108, 2013

3. Habibi S., Saleem M.A., Ramanan A.V. Juvenile Systemic Lupus Erythematosus: Review of Clinical Features and Management, Indian Pediatrics 48:879-887, nov 2011

4. Hoffman I.E., Lauwerys B.R., De Keyser F., Huizinga T.W., Isenberg D., Cebecauer L., et al. Juvenile-onset systemic lupus erythematosus: different clinical and serological pattern than adult-onset systemic lupus erythematosus. Ann Rheum Dis. 2009; 68:412-5.

5. Carreno L., Lopez-Longo F.J., Monteagudo I., Rodríguez Mahou M., Bascones M., González C.M., et al. Immunological and clinical differences between juvenile and adult onset of systemic lupus erythematosus. Lupus. 1999;8:287-92. ference between adult and pediatric onset lupus patients regarding prevalence of ANA, but elevated levels of anti-dsDNA antibodies are more common in pSLE than in aSLE. Low C3 levels also appear to be more prevalent in childhood-onset lupus.

Conflict of interest: none declared Financial support: none declared

6. Rina Mina, Hermine I. Brunner, Pediatric Lupus - Are There Differences in Presentation, Genetics, Response to Therapy, Damage Accrual Compared to Adult Lupus?, Rheum Dis Clin North Am. 2010 Feb; 36(1): 53-80.

7. Bertsias G.K., Tektonidou M., Amoura Z., et al. European League Against Rheumatism and European Renal AssociationEuropean Dialysis and Transplant Association Joint European League Against Rheumatism and European Renal AssociationEuropean Dialysis and Transplant Association (EULAR/ERA-EDTA) recommendations for the management of adult and paediatric lupus nephritis. Ann Rheum Dis. 2012; 71:1771-1782.

8. Eva D. Papadimitraki; David A. Isenberg, Childhood - and adult - onset lupus: an update of similarities and differences, Expert Rev Clin Immunol. 2009; 5(4):391-403.

9. Deborah M. Levy, Sylvia Kamphuis: Systemic Lupus Erythematosus in Children and Adolescents, Pediatr Clin North Am 2012 Apr; 59(2):345-364 